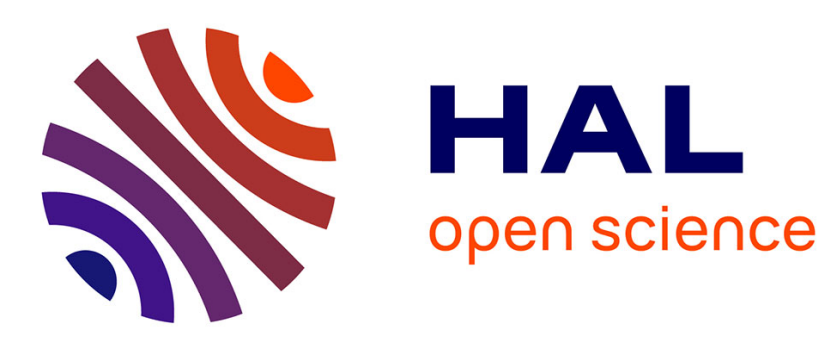

\title{
La tragédie parodiée ou les formes en combat
}

Roxane Martin

\section{To cite this version:}

Roxane Martin. La tragédie parodiée ou les formes en combat. Jeux et Enjeux des théâtres classiques (XIXe-XXe siècles), Mar 2001, Paris, France. pp.113-123. hal-02482757

\section{HAL Id: hal-02482757 \\ https://hal.univ-lorraine.fr/hal-02482757}

Submitted on 19 Feb 2020

HAL is a multi-disciplinary open access archive for the deposit and dissemination of scientific research documents, whether they are published or not. The documents may come from teaching and research institutions in France or abroad, or from public or private research centers.
L'archive ouverte pluridisciplinaire HAL, est destinée au dépôt et à la diffusion de documents scientifiques de niveau recherche, publiés ou non, émanant des établissements d'enseignement et de recherche français ou étrangers, des laboratoires publics ou privés. 
Cet article a été publié dans Littératures classiques, "Jeux et Enjeux des théâtres classiques $\left(\mathrm{XIX}^{\mathrm{e}}-\mathrm{XX}^{\mathrm{e}}\right.$ siècles)», Actes du colloque tenu en Sorbonne les 2 et 3 mars 2001, textes réunis et présentés par M. Bury et G. Forestier, Paris, Honoré Champion, $n^{\circ} 48$, printemps 2003, p. 113-123.

\section{La tragédie parodiée, ou les formes en combat ${ }^{1}$}

La critique littéraire a généralement tendance à interroger l'histoire en des termes duels. Classicisme et romantisme, tragique et comique, théâtres populaire et élitiste, genres «nobles » et «bas» sont autant de couples antagonistes indispensables pour découper des tranches d'histoire, marquer déclins et apogées et assigner à chaque siècle un genre littéraire prédominant. Suivant un tel procédé, le théâtre français du XIX ${ }^{\mathrm{e}}$ siècle se résume aisément à la fameuse bataille d'Hernani qui opposa, au Théâtre-Français, classiques et romantiques, «barbus » et "chevelus », libéraux et royalistes, mêlant largement littérature et politique. Entre une tragédie à bout de souffle et le drame triomphateur, la rupture entre deux esthétiques semblait être le fruit de l'histoire: "Êtesvous classique ou romantique? ", demandait Lousteau au jeune Lucien de Rubempré, "Mon cher, vous arrivez au milieu d'une bataille acharnée, il faut vous décider promptement. La littérature est partagée en plusieurs zones; mais nos grands hommes sont divisés en deux camps $»^{2}$. Ainsi Balzac caricaturait-il le milieu littéraire de la fin de la Restauration. Mais si le débat entre classiques et romantiques illustre assez bien les luttes politiques et l'accession des auteurs à la notoriété littéraire, il est de peu de secours pour le théoricien qui souhaite cerner leur contexte alors

1. Cet article a été publié dans Littératures classiques, «Jeux et Enjeux des théâtres classiques $\left(\mathrm{XIX}^{\mathrm{e}}-\mathrm{XX}^{\mathrm{e}}\right.$ siècles) », Actes du colloque tenu en Sorbonne les 2 et 3 mars 2001, textes réunis et présentés par M. Bury et G. Forestier, Paris, Honoré Champion, $\mathrm{n}^{\circ} 48$, printemps 2003, p. 113-123.

2. H. de Balzac, Illusions perdues, rééd. Paris, Gallimard, coll. " Folio », 1997, p. 253. 
que les codes d'écriture théâtrale et romanesque sont en pleine mutation.

L'un des caractères principaux du romantisme est en effet dans l'interpénétration des genres. Or poser la question d'une permanence du modèle classique au $\mathrm{XIX}^{\mathrm{e}}$ siècle oblige à faire éclater le cadre obligé des antagonismes frontaux pour inscrire la réflexion dans un système plus large qui prendrait en compte les relations des différentes formes théâtrales entre elles, du côté aussi bien des théâtres privilégiés que des scènes populaires. La parodie semble de ce point de vue le genre le plus propice à l'analyse : "Loin de se définir comme une forme de nihilisme, elle est le creuset d'où émergent de nouveaux genres, de nouvelles formes, ou encore de nouvelles perspectives théoriques ${ }^{1}$, relèvent avec justesse M. Espagne et M. Werner. En effet, devenue au XVIII siècle l'arme des auteurs de la Foire contre les Privilèges des Comédiens Français, la parodie met en œuvre un type d'écriture qui, après l'édit libérateur de 1791, a préludé au renouvellement des genres dramatiques. Une analyse des rapports entretenus entre la parodie et la tragédie, de la Révolution jusqu'à la fin de la monarchie de Juillet, fournit ainsi de précieux renseignements sur les modes de survivance et de réception du genre tragique à l'époque romantique. Tandis que les procédés d'écriture des parodies de la Lucrèce (1792) d'Arnault ou de l'Hector (1809) de Luce de Lancival perpétuent ceux d'un Fuzelier un siècle plus tôt, les parodies du Marino Faliero (1829) de Delavigne ou de la Lucrèce (1843) de Ponsard proposent quant à elles une étonnante réflexion sur la répartition et le mélange des genres. Laboratoire qui confronte le drame, le mélodrame et la tragédie, la parodie est devenue partie prenante dans la théorisation des genres, et ouvre des perspectives neuves sur l'affrontement entre classiques et romantiques.

\section{LE DIFFICILE STATUT DE LA PARODIE}

Née avec la littérature, la parodie a toujours eu du mal à s'inscrire dans les taxinomies génériques. À l'origine de cette difficulté une phrase de la Poétique d'Aristote:

1. M. Espagne, M. Werner, " Parodie et Sécularisation : à propos du mode parodique chez Heine et les jeunes hegeliens", dans Dire la Parodie, Colloque de Cerisy, New York, Peter Lang Publishing, 1989, p. 157. 
Homère a par exemple représenté ses personnages en mieux, Cléophon à l'identique, Hégémon de Thasos, le premier à avoir composé des parodies, et Nicharès, l'auteur de la Deiliade, en pire. $^{1}$

Ce bref passage, première occurrence connue du terme " parodie », a naturellement conduit structuralistes et poéticiens à s'interroger sur la place de la parôdia dans le système aristotélicien de répartition des genres ${ }^{2}$. La classification aristotélicienne, rappelons-le, est fondée sur une distinction entre deux types d'action (basse ou noble) et deux modes de représentation (narratif ou dramatique). Du croisement de ces deux oppositions résulte une grille à quatre cases dont trois ont été clairement définies par Aristote, la tragédie étant la représentation d'une action noble en mode dramatique; l'épopée, d'une action noble en mode narratif; la comédie, d'une action basse en mode dramatique. La quatrième case du système, caractérisée par le croisement de l'action basse et du mode narratif, n'est pas explicitement déterminée par le philosophe. Seule la référence à des œuvres plus ou moins directement désignées sous le terme de parôdia invite à faire correspondre la parodie à la catégorie générique de l'épopée d'action basse.

Toutefois, l'hypothèse d'une parodie relevant exclusivement du narratif est loin de s'appliquer à l'ensemble de la production poétique de l'Antiquité. Hégémon de Thasos, par exemple, seul auteur auquel Aristote rattache expressément la parôdia, est plus connu pour ses parodies dramatiques. Finalement, seuls la Deiliade - que l'on suppose d'après son étymologie une Iliade des lâches (deilos signifiant «lâche»), une anti-épopée en somme - et le Margitès, auquel Aristote fait référence au quatrième chapitre de son traité, vérifient l'organisation quadripartite du système : «... le rapport que l'Iliade et l'Odysée entretiennent avec les tragédies est analogue au rapport qu'entretient le Margitès avec les comédies » ${ }^{3}$ précise-t-il. En effet, aujourd'hui disparu et longtemps attribué à Homère, le Margitès, du nom de son héros, narrait les aventures d'une sorte d'anti-Ulysse dont la moindre initiative tournait à la catastrophe. Mais ces considérations s'avèrent peu concluantes quant au statut générique de la parodie : en réduisant les fictions à leur seule fonction représentative, Aristote propose un système qui

1. Aristote, Poétique, 1448a, trad. M. Magnien, Paris, LGF, 1990.

2. Voir à ce sujet l'ouvrage de G. Genette, Palimpsestes, chap. 3, Paris, Seuil, 1982, rééd. coll. « Points essais », 1991.

3. Aristote, Poétique, 1449a, op. cit. 
non seulement exclut toute poésie non figurative, mais propose un nombre de critères insuffisant pour évaluer un genre caractérisé d'abord, conformément à l'étymologie, comme para ôdè, " chant d'à-côté », c'est-à-dire dans lequel l'imitation prend pour objet non plus proprement le réel mais une imitation première.

Le simple fait de poser la parodie comme imitation demeure lui-même problématique. En effet, comme le souligne G. Genette :

la parodie et le travestissement $[\ldots]$ ne peuvent en aucun cas être définis comme des imitations, mais bien comme des transformations, ponctuelles ou systématiques, imposées à des textes. ${ }^{1}$

Si la parodie dans l'Antiquité semble avoir désigné un genre ${ }^{2}$, elle correspondait aussi à une certaine technique de citation ainsi définie par Quintilien : «Forger des vers qui ressemblent à des vers connus, [c'est] ce que l'on nomme parodie $»^{3}$. La perte ou l'absence dans la Poétique d'un développement consacré à la parôdia a contribué à rattacher la parodie à la rhétorique et à la définir restrictivement comme une pratique de la citation détournée. Lorsqu'il aborde le sens qu'elle adopta à l'âge classique, D. Sangsue se voit contraint d'admettre que

même Boileau, qui a parodié Le Cid dans Chapelain décoiffé, ne traite pas de la parodie. Considérée comme une opération limitée sur les textes, elle est censée appartenir au domaine des tropes. ${ }^{4}$

Forts de ces constats — une parodie hypothétiquement définie comme transposition de l'épopée dans le registre du «bas» et simple opération de recontextualisation de vers connus - les théoriciens actuels peuvent difficilement accorder à la parodie un statut de genre littéraire. En distinguant le pastiche de la parodie sur ce que l'un imite et l'autre transpose, G. Genette suit la

1. G. Genette, Palimpsestes, op. cit., p. 111.

2. C'est tout au moins la théorie avancée par M. Bakhtine qui voit une institutionnalisation de la parodie à travers le couple tragédie/drame satyrique. Voir L'CEuvre de Francois Rabelais et la culture populaire au Moyen Age et sous la Renaissance, trad. A. Robel, Paris, Gallimard, 1970, rééd. coll. «Tel », 1982.

3. Quintilien, De l'institution oratoire, trad. J. Cousin, Paris, Les Belles Lettres, 1975, Livre I, VI, 3, p. 98.

4. D. Sangsue, La Parodie, Paris, Hachette, 1994, p. 17. Le Chapelain décoiffé est une œuvre écrite en collaboration par Boileau, Chapelle, Furetière et Racine en 1665. 
conception classique selon laquelle l'acte parodique vise à recontextualiser un fragment de texte connu : le Chapelain décoiffé se fait ainsi le parangon d'une parodie perçue au sens strict comme «transformation ludique d'un texte singulier». Il ne saurait donc exister de parodie de genre puisque, nous rappelle encore l'auteur de Palimpsestes, " imiter, c'est généraliser » ${ }^{1}$, c'est-à-dire s'en prendre aux règles, au style caractérisant un genre, ce qui appartient en propre au pastiche.

Toutefois, l'historien, et plus encore l'historien du théâtre, peut difficilement récuser l'évidence d'un lien intime au cours du $\mathrm{XVIII}^{\mathrm{e}}$ siècle entre parodie et tragédie. Ainsi M. de Rougemont au seuil d'un article récent sur le genre:

La parodie théâtrale apparaît comme genre constitué au XVIII ${ }^{\mathrm{e}}$ siècle en France. [...] L'opéra est la première cible ; ce seront ensuite les tragédies individuelles, puis le genre lui-même. ${ }^{2}$

En effet, le XVIII ${ }^{\mathrm{e}}$ siècle, sous l'impulsion des auteurs de la Foire, a élargi la notion de parodie : de simple procédé rhétorique elle se constitue en genre, s'institutionnalise, et initie, son dédoublement structurel et la mise à nu de ses ficelles de fabrication aidant, une discussion critique sur les conventions littéraires. Exclusivement attachée à la tragédie dans un premier temps, elle s'étend progressivement à toutes les formes théâtrales, y compris ellemême ${ }^{3}$, et s'impose, à l'aube du XIX ${ }^{e}$ siècle, en tant qu'instrument de théorisation des genres.

Ainsi, la définition de la parodie diffère selon le contexte littéraire considéré. L'historien du théâtre la considère volontiers comme un genre autonome, là où le poéticien cherche avant tout à dissiper toute confusion en la restreignant à sa fonction première de détournement textuel. Notion complexe et exposée à l'amalgame, la parodie a beau peiner à s'imposer dans les taxinomies génériques, elle n'en demeure pas moins intimement liée à la notion de genre littéraire. Miroir d'une ouvre singulière dont elle abolit l'unicité et dénude les processus de fabrication, elle s'offre à la littérature comme moyen de réflexion sur ces principes de production. Le $\mathrm{XIX}^{\mathrm{e}}$ siècle, qui a vécu une redéfinition

1. G. Genette, op. cit., p. 111

2. M. de Rougemont, "Le rire de la parodie: Freud ou Bergson?", Dix-Huitième siècle, «Le Rire », n³2, 2000, p. 51.

3. On citera par exemple les Huit Mariamnes de Piron, parodie des Quatre Mariamnes de Fuzelier, elles-mêmes parodie de la Mariamne de Voltaire. 
complète du concept de littérature, lui a dévolu un rôle prépondérant dans la redistribution des codes d'écriture. C'est la raison pour laquelle la parodie théâtrale nous invite à un regard radicalement différent sur la mutation de l'esthétique théâtrale au siècle « romantique ».

\author{
« MIROIR, Ô MON BEAU MIROIR... » : LE RAPPORT \\ Parodie/Tragédie SOUS La RÉvOlution ET L'EMPIRE
}

Si l'édit de 1791 a provisoirement libéré le théâtre de ses privilèges, les pratiques d'écriture n'ont pas pour autant suivi. En effet, la tragédie, tout en renonçant aux héros «nobles », continue de remporter les suffrages, tandis que la Foire maintient à l'affiche ses pantomimes d'antan. Le véritable bouleversement réside alors dans l'élargissement du public et dans la reconnaissance d'un théâtre populaire jusque là confiné aux baraques foraines. Aussi, si la censure et le contrôle policier eurent vite fait de freiner l'élan créatif des quelques dizaines de salles qui s'ouvrirent soudain, ils ne purent cependant empêcher le théâtre de s'inscrire, cette fois de façon irréversible pour tout le siècle à venir, au centre de toute la sociabilité urbaine. Dès lors, l'ancienne partition des genres ne pouvait qu'apparaittre caduque : les techniques d'écriture mises au point par la Foire tout au long du XVIII ${ }^{\mathrm{e}}$ siècle s'imposaient alors qu'allaient voir le jour de nouveaux genres dont la grandiloquence spectaculaire bouleversait les présupposés d'écriture reçus. Mais la dichotomie populaire/élitiste ne disparut pas pour autant. La vénération du gouvernement révolutionnaire puis de l'Empire pour la tragédie contribua à maintenir la ligne de partage entre un théâtre censé "sérieux» et une production populaire tout juste bonne à divertir les classes « laborieuses». Aussi, sous le poids des contraintes et des restrictions retrouvées, les auteurs du Boulevard conservèrent la même perception de la tragédie: un modèle éprouvé mais contraignant qui méritait d'être mis à distance. Le rapport parodie/tragédie persista donc pendant les premières années du XIX ${ }^{\mathrm{e}}$ siècle tel qu'auparavant.

Une rapide analyse des parodies de la période révolutionnaire et impériale permet de repérer les procédés de la parodie théâtrale française, que les vaudevillistes mettront à leur tour en œuvre à l'encontre du drame. Deux fonctions essentielles caractérisent la parodie théâtrale : la première en rapport avec la nécessaire 
filiation entre parodie et parodié, la seconde avec la critique des conventions du genre mis en cause. La filiation - la reconnaissance en filigrane de l' "hypotexte» pour reprendre la terminologie genettienne - se reconnaît à deux moyens principaux: la recontextualisation du sujet et la mécanisation des procédés d'écriture. La mécanisation consiste à ne retenir que les grandes articulations de l'intrigue originelle sous forme réduite qui n'accorde plus à la langue l'espace nécessaire à sa poéticité native. La recontextualisation, quant à elle, consiste à dépayser la fable dans un cadre insolite contrastant avec le texte-cible de manière à donner corps au burlesque.

Ont recours à ces divers procédés: Le Projet manqué, on Arlequin taquin ${ }^{1}$, parodie de la Lucrèce (1792) d'Arnault; Médard, fils de Gros-Jean', parodie d'Oscar, fils d'Ossian (1796) du même ; Hector, ou le valet de carreau ${ }^{3}$, parodie de l'Hector (1809) de Luce de Lancival; Mabomet Barbe-blene, on la Terreur des ottomanest, parodie de Mahomet II (1811), ou encore Omazette, on Jozet en Champagne, parodie d'Omasis, ou Joseph en Egypte (1806) de Baour de Lormian. Cette dernière, par exemple, reprend les révélations de Joseph qui, devenu ministre de Pharaon sous le nom d'Omasis, cherche avant d'épouser sa fille Almaïs à lever les voiles d'un passé obscur en renouant avec sa propre famille. Toutefois, le palais de Pharaon devient la cour (de ferme bien-sûr) de Monsieur Farinon; le terrible Rhamnès, instigateur du meurtre d'Omasis, se change en l'insolite Raflès, et Almaïs, dont le rôle dans la tragédie reste plutôt discret, s'impose sous la plume des vaudevillistes sous le nom d'Inutilis. Ainsi, par ces deux procédés, la parodie provoque une dissonance entre fond et forme génératrice de comique. Si la recontextualisation tend, par son jeu sur les contrastes, à vider les thèmes de leur valeur archétypale, la mécanisation dévitalise l'œuvre en réduisant la forme à de simples ficelles. De tels décalages permettent donc de rendre apparents les procédés de

1. Parodie en un acte de Barré, Radet et Desfontaines, jouée au Vaudeville en mai 1792.

2. Parodie en deux actes de Gouffé et Rouhier-Deschamps, jouée au Théâtre de la Cité en juillet 1796.

3. Jeu de cartes en cinq parties, par Désaugiers, Balisson de Rougemont et Gentil, joué au Vaudeville en février 1809.

4. Imitation burlesque en un acte de Chazet, Merle et Ourry, jouée aux Variétés en mars 1811.

5. Parodie vaudeville en un acte de Barré, Radet, Desfontaines et Dieulafoy, jouée au Vaudeville en octobre 1806. 
fabrication de l'œuvre: en rompant l'illusion, la parodie anéantit l'autorité du modèle et met en place une véritable discussion critique sur les procédés d'écriture qui lui sont propres.

De la Révolution à l'Empire, trois points d'attaque reviennent inlassablement dans la quinzaine de parodies de tragédies. Le premier touche évidemment aux unités, et particulièrement à l'unité de lieu. Il est vrai que depuis le drame bourgeois le système des unités est le premier visé dans les tentatives pour ébranler l'édifice classique. Ainsi, lorsque la tragédie s'octroie la liberté de changements de décors, la parodie ne manque pas de relever cette entorse aux conventions, telle Lucrèce qui, dans Le Projet manqué, on Arlequin taquin, s'étonne, non sans humour :

La scène change !... Eh mais, pourquoi ?

Comme je me trouve chez moi!

Cette chambre est la mienne...

Cela me donne du souci...

\section{(En soupirant)}

Puisse-t-on ne violer ici

Que l'unité de scène !

Le deuxième point à exciter la verve des parodistes concerne les récits relatifs aux actions extra-scéniques - comme la tentative de meurtre menée contre Omasis qui donne lieu dans la parodie à une scène désopilante - et les monologues d'exposition. Mais le coup de bélier le plus puissant contre la forteresse tragique porte sur l'alexandrin. Aussi est-il courant qu'un vers de Racine s'égare dans le discours d'un des personnages - c'est le cas par exemple dans Médard, fils de Gros-Jean -, ou encore que le héros de la parodie se mette subitement à parler en vers, comme Omazette au cours de la première scène :

\section{OMAZETTE}

«L'âge de leurs ayeux [sic] touche au berceau du monde ».

HAZARDEL

Comment l'âge touche... Qu'est-ce que cela veut dire ?

OMAZETTE

1. Barré, Radet, Desfontaines, Le Projet manqué, on Arlequin taquin, Paris, Brunet, 1792, sc. 8, p. 27. 
Cela veut dire qu'ils sont gentilshommes normands, et moi aussi. Les fils du grand Jaco... la plus belle race de l'univers. ${ }^{1}$

Toutefois, en dépit de ces attaques, la parodie maintient des rapports relativement cordiaux à l'égard du genre aîné. Le vaudeville final atteste toujours la bonne entente entre parodiste et parodié, confirmant l'hypothèse selon laquelle la parodie contribue au succès de l'œuvre qu'elle tourne en dérision. Ainsi Hector, ou le valet de carreau peut-il finir sur ces mots :

Venez chanter et rire,

À la mort

De notre Hector,

Et retournez le lendemain

Applaudir au chantre divin

De l'Hector du voisin. ${ }^{2}$

Le discours de la parodie fut au contraire plus acrimonieux à l'égard du drame. C'est le constat dressé par M. de Rougemont qui, citant les vaudevilles finaux de Marionnette et d'Harnali ou la contrainte par $c^{3}{ }^{3}$, s'étonne d'une âpreté de ton sans précédent dans la parodie théâtrale du XVIII ${ }^{\mathrm{e}}$ siècle ${ }^{4}$. Les tragédies parodiées sous la monarchie de Juillet témoignent d'une même aigreur envers les romantiques. Les cinq années de suppression de la censure qui marquèrent le début du règne de Louis-Philippe contribuèrent en effet à abolir la frontière entre scènes du Boulevard et théâtres privilégiés. La rencontre désormais facilitée entre les formes et les répertoires permit au théâtre de consolider une esthétique, en germe depuis le début du siècle, fondée sur le mélange des genres. Dès lors, le regard du Boulevard à l'égard de la tragédie changea radicalement: déboulonnée par les invectives des romantiques, celle-ci devenait une alliée indispensable contre le nouveau prétendant à la primauté littéraire qu'était devenu le drame romantique.

1. Barré, Radet, Desfontaines, Dieulafoy, Omazette, ou Jozet en Champagne, Paris, Masson, 1807, sc. 1, p. 6.

2. Balisson de Rougemont, Désaugiers, Gentil, Hector, ou le valet de carreau, Paris, Masson, 1809, p. 38.

3. Parodies de Marion de Lorme et d'Hernani.

4. M. de Rougemont, art. cit. 


\section{LA TRAGÉDIE PARODIÉE SOUS LA MONARCHIE DE JUILLET : UN MÉTATEXTE DU DRAME}

S'il est difficile de théoriser la forme parodique, c'est sans doute qu'elle remet en cause la doxa de l'imitation. De même, lorsque les romantiques eurent à proposer une forme théâtrale en rapport avec une esthétique du mélange, de l'alliage et de l'harmonie des contraires, la nécessité s'imposa de rompre avec la doctrine de l'imitation de l'Antiquité. Ainsi, en cherchant à affermir les contours d'un genre qui se devait de conjuguer le «drame de la vie» et le «drame de la conscience», le destin individuel et l'Histoire d'un peuple, ils ressentirent le besoin d'attaquer la tragédie dans toutes ses conventions. Soutenu par des manifestes tels que la Préface de Cromwell de Victor Hugo et le Racine et Shakespeare de Stendhal, le drame consolida ses fondations en subvertissant les anciens codes: transgression des unités de temps et de lieu, abolition de la séparation du comique et du tragique, et déstructuration de l'alexandrin. En se faisant critique à l'égard du moule classique pour le dépasser, le drame recourut luimême, d'une certaine façon, à une écriture de type parodique. Cette hypothèse, qui peut paraittre quelque peu hâtive et audacieuse, fut pourtant celle que soutinrent les auteurs de parodies. C'est ainsi que dans Lucrèce à Poitiers, on les Écuries d'Augias, parodie de la Lucrèce de Ponsard, par exemple, Brutard, directeur de théâtre au bord de la faillite en quête d'un genre de spectacle susceptible d'attirer le public, pouvait s'exclamer, après avoir auditionné Guanhumara, allégorie du drame :

\section{BRUTARD}

Ô ciel ! nous faire entendre un semblable blasphème !

\section{MIGNOT}

Quelle gaillarde, oh! oh!

\section{PATRARQUE}

Je dois en être blême !

MIGNOT

Ah ! ma foi, ce n'est pas avec ça que jamais, Mon pauvre directeur, vous comblerez vos frais... 


\section{BRUTARD}

Non... l'espérer serait faire une erreur énorme ; Les chevaux qu'aujourd'hui j'ai mis à la réforme Conviendraient encor mieux...

\section{MIGNOT}

Que vous soyez tenté par un genre si bas, Que vous veuillez enfin jouer la parodie...

BRUTARD

La parodie !... Eh ! mais, c'est une tragédie.

\section{MIGNOT}

Ah bah!

$$
\text { PATRAQUE }
$$

Bah!

\section{BRUTARD}

\section{Ces messieurs la comprennent ainsi $!^{1}$}

Si le drame se définissait en prenant le contre-pied des conventions de la tragédie, la parodie n'avait plus qu'à utiliser la forme tragique afin d'atteindre le drame. Un nouveau type de parodie théâtrale s'esquissait dès lors. Abandonnant la fonction de simple miroir qui, suivant les principes de recontextualisation et de mécanisation, lui permettait de jeter un regard critique sur les conventions du genre dont relevait le texte cible, la parodie s'engageait sur le terrain de la théorisation des genres en proposant une histoire littéraire sous une forme dramatisée.

La majeure partie des tragédies parodiées, sous la monarchie de Juillet, illustre, en effet, la querelle entre classiques et romantiques. Si la parodie du drame continuait à fonctionner sur les modes de la parodie de type "miroir», celle de la tragédie abondait en réflexions méta-narratives de toutes sortes tendant à dénoncer sa prétention à l'innovation, qui plus que tout, semble-til, indisposait les auteurs du Boulevard à l'égard du drame. Il est vrai aussi que depuis une trentaine d'années les genres secondaires pratiquaient allègrement le mélange des genres et transgressaient

1. [Chapelle], Léonard, Lucrèce à Poitiers, on les Écuries d'Augias, tragédie mêlée de vaudevilles jouée au Gymnase Dramatique à partir du $1^{\text {er }}$ juin 1843, Paris, Furne et Cie éd., Tresse, Marchant, 1843, sc. 5, p. 7. 
les unités. Aussi, dès 1829, année qui vit le drame pénétrer l'antre de Melpomène et la Porte-Saint-Martin accueillir la tragédie, les hostilités à l'égard du drame commencèrent : «... Une rupture... une révolution, du scandale... voilà ce que j'aime. Oh! du scandale... en littérature, il faut toujours prendre ça, faute de mieux $»^{1}$ s'exclamait M. Dujour dans Marino Faliero à Paris. Une constante des parodies de tragédie sous la monarchie de Juillet fut en effet de crier à l'usurpation. Dans Le Doge, et le dernier jour d'un condamné, ou le Canon d'alarme, ou les classiques et les romantiques, autre parodie du Marino Faliero, le Dernier jour d'un condamné, personnage allégorique du romantisme, dévoilait ainsi le mode de fabrication d'un drame :

Vous composez un sombre Mélodrame,

Et vous courez le lire au Boulevard.

On le refuse ; un espoir vous enflamme,

C'est qu'il pourra briller un peu plus tard!

Vous retouchez le chef-d'œuvre historique,

Certain qu'ailleurs il peut trouver accès,

Et qu'il doit même obtenir un succès :

S'il n'est pas bon pour l'Ambigu-Comique,

Il va de droit au Théâtre-Français ! ${ }^{2}$

En revendiquant de la sorte l'assimilation du drame et du mélodrame, la parodie soulevait l'épineuse question de la notion de genre en relation avec le tragique. Placer le drame, le mélodrame et la tragédie sur le même plan revenait à ruiner les fondations théoriques du drame et réhabilitait la tragédie tout en légitimant les formes populaires. Ainsi, tous les fondements théoriques du drame furent tournés en dérision, à commencer par la refonte du vers.

Dans Le Doge, en effet, Clair de Lune, jeune-fille déchirée dans son éducation littéraire entre les volontés de son père, M. de Vieux-Vers, partisan du classicisme, et les penchants de sa mère pour les théories romantiques, prétendait : «Vous serez satisfaits tous les deux. (À son père) J'ai traduit les Lettres à Atticus. (À sa mère) Je traduis aussi Les Orientales, je commence à les expliquer assez

1. Bayard, Varner, Marino Faliero à Paris, folie à-propos vaudeville en un acte, Théâtre du Vaudeville, 7 mai 1829, Paris, Olivier \& Barba, 1829, sc. 8, p. 21.

2. [Brazier], Simonnin, Vanderburch, Le Doge, et le dernier jour d'un condamné, ou le Canon d'alarme, ou les classiques et les romantiques, vaudeville en trois tableaux, Théâtre des Nouveautés, 20 mai 1829, Paris, Quoy, 1829, sc. 18, p. 34. 
bien $»^{1}$. De la même façon, Patraque, dans Lucrèce à Poitiers, admirateur du vers romantique, s'écriait : «On dirait de la prose ou les vers se sont mis! $»^{2}$. En caricaturant de la sorte le vers romantique, la parodie procédait à une inversion radicale des formes classique et romantique. Si le drame était encensé par le Théâtre-Français, la tragédie, jouée sur le Boulevard, devenait bien plus « romantique » que le drame. Ainsi une scène du Doge pouvaitelle caricaturer :

\section{LE DERNIER JOUR}

Soyez le bienvenu ; mais pourrions-nous savoir, Qui nous procure ici le bonheur de vous voir?

LE DOGE, avec une dignité comique.

Victime des abus ou l'intrigue l'entraîne, J'ai quitté pour jamais l'injuste Melpomène, Et je n'ai fait qu'un saut, dans ce triste destin, De la Scène-Française à celle Saint-Martin.

\section{FIGARO}

Contez-nous donc, seigneur, vos nobles infortunes ; Car le grand Doge ici ne vient pas pour des prunes. Pour payer le trajet, aviez-vous du quibus?

\section{LE DOGE}

Je n'avais que cinq sous, $j$ 'ai pris un Omnibus.

\section{Clair DE LUNE}

Un Doge en Omnibus! Un Doge qui partage, Des badauds de Paris, le modeste équipage!

$\mathrm{Ah} !$ que c'est romantique ! et que c'est vaporeux $!^{3}$

De nombreux autres exemples de critiques du drame par la tragédie parodiée pourraient être invoqués, dont il ressort une certaine vénération du Boulevard pour les auteurs classiques. On eût pu s'attendre en effet que ce dernier fit meilleur accueil à des conceptions théâtrales imposant sur la scène du Théâtre-Français des techniques d'écriture qu'il avait lui-même fortement contribué à façonner, et que la parodie, qui va en général avec le succès, encensât le drame à ses premiers triomphes et le condamnât aux

1. Le Doge, et le dernier jour d'un condamné, op. cit., sc. 5, p. 13.

2. Lucrèce à Poitiers, op. cit., sc. 4, p. 6.

3. Le Doge, op. cit., sc. 18, p. 32-33. 
premières difficultés. Mais il n'en fut rien : du Henri III (1829) de Dumas aux Burgraves (1843) de Hugo, les vaudevillistes redoublèrent leurs attaques contre le genre «romantique». Toutefois, ce qu'ils semblent avoir condamné n'est pas tant la forme dramatique par elle-même que ses assises théoriques. Ainsi, en confrontant la jeune école au modèle ancien, la parodie niait l'aspect novateur du drame, et s'imposait elle-même dans le débat sur la théorisation des genres, pour le plus grand profit de l'historien du théâtre.

Tandis qu'elle se bornait au XVII ${ }^{\mathrm{e}}$ siècle à une technique de citations détournées, éventuellement dans les querelles littéraires comme ce fut le cas du Chapelain décoiffé, elle illustra au XVIII ${ }^{\mathrm{e}}$ siècle la lutte entre la Foire et les théâtres privilégiés. Tragédie et opéra ayant suffisamment affermi leurs codes, la parodie pouvait à son tour s'ériger en genre, et s'en prendre, par le biais des transformations imposées à un texte unique, à l'ensemble des conventions du genre auquel il appartenait. Si l'on s'en tient pour la parodie théâtrale du XIX siècle aux seuls exemples des Harnali, ou la contrainte par cor, Cornaro, tyran pas doux, ou Les Hures-Graves, on peut croire à une continuité entre les $\mathrm{XVIII}^{\mathrm{e}}$ et $\mathrm{XIX}^{\mathrm{e}}$ siècles. La lecture des tragédies parodiées sous la monarchie de Juillet révèle l'existence d'un autre type de parodie qui, certes, peut difficilement prétendre à l'épithète "parodique » selon la théorie genettienne, mais n'en montre pas moins que le théâtre romantique français ne saurait être réduit au seul drame. En inventant une forme théâtrale qui fait se côtoyer, en manière d'allégorie, la totalité des genres dramatiques de l'époque, elle récuse en effet le système binaire qui prévaut dans la réception moderne du $\mathrm{XIX}^{\mathrm{e}}$ siècle, et propose une tout autre définition du concept de «mélange des genres». La critique du drame romantique dans les parodies de tragédies atteste que la rupture en matière d'esthétique s'est signalée d'abord dans l'abandon du manifeste littéraire en tant que soutien théorique. C'est du moins le schéma interprétatif qu'aura tenté d'esquisser la présente analyse.

Roxane MARTIN 